\title{
Effect of Ligustrazine Nanoparticles on Th1/Th2 Balance by TLR4/MyD88/NF-KB Pathway in Rats with Postoperative Peritoneal Adhesion
}

\section{Lili Yang}

Nanjing University of Chinese Medicine

Ziyu Lian

Nanjing University of Chinese Medicine

Bin Zhang

Ningbo Hospital of traditional Chinese medicine

Zhengjun Li

University of St Andrews

\section{Li Zeng}

Nanjing University of Chinese Medicine

\section{Wenlin Li}

Nanjing University of Chinese Medicine

\section{Yaoyao Bian ( $\sim$ bian@njucm.edu.cn )}

Nanjing University of Chinese Medicine https://orcid.org/0000-0002-9968-2501

\section{Research article}

Keywords: Postoperative peritoneal adhesion, ligustrazine nanoparticle, Th1/Th2 balance, TLR4/MyD88/NF-kB pathway

Posted Date: May 27th, 2020

DOI: https://doi.org/10.21203/rs.3.rs-26682/v1

License: (a) (1) This work is licensed under a Creative Commons Attribution 4.0 International License. Read Full License

Version of Record: A version of this preprint was published at BMC Surgery on April 26th, 2021. See the published version at https://doi.org/10.1186/s12893-021-01201-7. 


\section{Abstract \\ Background}

Postoperative peritoneal adhesion (PPA) is regarded as fibrous bands connecting both injured abdominal wall and organs or adjacent tissues and associated with T helper (Th) 1 and Th2 differentiation. However, the critical role of immunopathogenesis of adhesion formation was precisely unknown. Previously we demonstrated that ligustrazine have positive effects on adhesion formation. And microarray analysis revealed TLR4/MyD88/NF-KB pathway as an important underlying regulatory mechanism in the onset and development of PPA. To further explore the possible mechanism of PPA and enhance the specificity of ligustrazine delivery to injured sites of rodent models, a new agent polylactic acid (PLA) nanoparticles loaded with ligustrazine, that is, ligustrazine nanoparticles (LN) were applied.

\section{Methods}

Twenty-four SD rats were randomly divided into sham, model, $\mathrm{LN}$ and sodium hyaluronate (SH) groups. The structure of LN, including entrapment efficiency (EE) and loading capacity (LC), and in vitro drug release were calculated. Adhesions were scored and the masson's trichrome staining was used to determine the collagen deposition. The expression of TLR4, MyD88 and NF-KB were measured by qPCR, Immunohistochemistry and western blot assay. Moreover, Th1-related cytokines (IFN- $\gamma$, IL-12), Th2-related cytokines (IL-4, IL-6) in the cecum tissue and serum were conducted by ELISA.

\section{Results}

LN had good EE, LC and control-release delivery characters with fairly uniform diameter and spherical morphology. It could effectively prevent adhesion formation after surgery. Besides, it could reduce collagen fibers accumulation, downregulate the expression levels of TLR4, MyD88 and NF-KB, and maintain Th1/Th2 balance.

\section{Conclusions}

Ligustrazine nanoparticles had effective effects on Th1/Th2 balance by regualtingTLR4/MyD88/NF-KB pathway in PPA rats. It may be served as a promising therapy on postoperative adhesion formation.

\section{Background}

Postoperative peritoneal adhesion (PPA) is regarded as fibrous bands connecting both injured abdominal wall and organs or adjacent tissues, which is an inevitable complication following any kinds of abdominal surgery. It remains one of the most challenging issues in surgery fields around the world. Approximately $50-85 \%$ patients who underwent abdominal surgery suffered from various adhesion- 
associated problems including abdominal pain, female infertility and intestinal obstruction ${ }^{[1][2]}$. In USA, about 117 per 100,000 people re-hospitalized due to the above complications, and the direct cost spent in adhesive complications reached up to $\$ 1.3$ billion ${ }^{[3][4]}$. It brought out a tremendous burden on public health care.

The formation of adhesion is a multistep process that related to oxidative stress ${ }^{[5]}$, inflammatory response and the fibrinolytic system ${ }^{[6]}$. However, the critical role of immunopathogenesis was precisely unknown. Few studies suggested that immune progress controlled by $T$ cells played prominent roles in adhesion formation ${ }^{[7][8]}$. T cells can be divided into two subpopulations, that is Thelper (Th) 1 and Th2 ${ }^{[9]}$. Mounting evidences indicated Th1/Th2 ratio would shift due to the major or minor abdominal surgery ${ }^{[10]}$ [11]. It is suggested that Th1 cells are the effector cells in mediating adhesion formation ${ }^{[12]}$. And a significant increase of Th2 cells was found in the adhesion area of a rodent experiment ${ }^{[8]}$. Previously, we reported that the exact roles of TLR4/MyD88/NF-KB signaling pathway in the pathogenesis of PPA based on the microarray analysis ${ }^{[13]}$. Herein, it might be a beneficial way to find new approach to regulate Th1/Th2 balance through TLR4/MyD88/NF-KB regulatory chain.

So far, due to the lack of definitive guidelines and reliable strategies for adhesion prevention and treatment ${ }^{[4][14]}$. It is urgent to understand the underlying mechanism of PPA and identify more effective treatment to prevent adhesion formation. Fortunately, with the development of biology and materials science, various anti-adhesion products such as biological scaffolds, membranes and drugs were applied on animals and in clinics for its prevention or decreasing of tissue adhesion ${ }^{[15]}$. Among of these strategies, nanoparticles with unique characteristic features were also widely used in adhesion formation ${ }^{[16]}$, peritoneal fibrosis ${ }^{[17]}$ and cancer ${ }^{[18]}$.

We previously reported that ligustrazine, a kind of bioactive components derived from the root of herbal Ligusticum Chuanxiong hort (Umbelliferae) had positive effects on adhesion formation in vivo and in vitro $^{[19][20]}$. However, the disadvantages of ligustrazine on injured sites i.e. rapid absorption and metabolism, short half-life and uneven distribution limited its application in biomedical fields. The effects of ligustrazine on pre-clinical studies are far from satisfactory. Recent study indicated that ligustrazinepolylactic acid (PLA) sustained-release microspheres might be a promising agent due to the characteristics of stable and sustained release effects on bioactive ingredients ${ }^{[21]}$. As PLA is emerging as an ideal drug carrier for its favorable biocompatibility and biodegradable ${ }^{[22]}$. To enhance the specificity of ligustrazine delivery to injured tissues, the new agent PLA nanoparticles loaded with ligustrazine, that is, ligustrazine nanoparticles $(L N)$, were used in our study. We aimed to explore the exact effects of $L N$ on PPA and identify the possible mechanism.

\section{Methods}

\section{Preparation and microstructure of $\mathrm{LN}$}


Ligustrazine $\left(\mathrm{C}_{8} \mathrm{H}_{12} \mathrm{~N}_{12}\right.$, purity $\left.\geq 98 \%\right)$ was provided by Tokyo Chemical Industry (Japan). The LN was prepared as our previous studies reported ${ }^{[23][24]}$. Appropriate amount of ligustrazine and PLA $(1: 4)$ were taken and dissolved in the acetone solution. The mass concentration of PLA in the acetone solution should be $20 \mathrm{~g} / \mathrm{L}$. This solution was called oil phase. $0.25 \%$ poloxamer solution with four times volume of the oil phase was used as the water phase. Then, the oil phase was poured rapidly into the water phase at a high agitation speed at $30{ }^{\circ} \mathrm{C}$, and the two phases were kept stir for 70 min until the acetone was evaporated. Finally, $1 \mathrm{mg} / \mathrm{mL}$ ligustrazine nanoparticles were got. One-drop sample of the above LN was dripped onto the copper grid with a thin carbon film. After the drop was air-dried, phosphotungstic acetate solution was applied to stain for $5 \mathrm{~min}$. The microstructure of $L N$ was visualized under the high resolution transmission electron microscope (HRTEM, Japan).

\section{Determination of entrapment efficiency (EE) and loading capacity (LC)}

$\mathrm{LN}$ was centrifuged at $25,000 \mathrm{r} / \mathrm{min}$ for $60 \mathrm{~min}$ and the supernatant was harvested to measure EE and LC. About $0.2 \mathrm{~mL}$ supernatant mixed with $4.8 \mathrm{~mL}$ methanol were used to calculate the contents of free ligustrazine in the supernatant under the high performance liquid chromatography (HPLC), which was regarded as $\mathrm{W}_{\mathrm{f}}$. And $0.2 \mathrm{~mL} \mathrm{LN}$ mixed with $0.5 \mathrm{~mL} \mathrm{~N}$, N-dimethylformamide and $4.3 \mathrm{~mL}$ methanol were applied to calculate the total amount of LN under HPLC, which was named as $\mathrm{W}_{\mathrm{t}}$. EE and LC were calculated as follows ${ }^{[25]}$ :

$E E(\%)=\left[\left(W_{t}-W_{f}\right) / W_{t}\right] \times 100 \%$

$\operatorname{LC}(\%)=\left[\left(W_{t}-W_{f}\right) / W_{n}\right] \times 100 \%$

Whereas, $W_{n}$ was the weight of $L N$ after lyophilization.

\section{In vitro drug release}

Due to ligustrazine was lipid-soluble drugs, phosphate buffer was used as release medium. The phosphate buffer was prepared as follows: $1.36 \mathrm{~g}$ potassium dihydrogen phosphate mixed with $79 \mathrm{~mL}$ $0.1 \mathrm{~mol} / \mathrm{L}$ sodium hydroxide solution, which were diluted in deionized water up to $200 \mathrm{~mL}$. Then the mixed solution was re-suspended with $0.25 \%$ poloxamer. $1 \mathrm{~mL} \mathrm{LZ}$ was added in a membrane dialysis bag in $30 \mathrm{~mL}$ above release medium at $\mathrm{PH}=7.4$ at room temperature with stirring constantly. During this time, $1 \mathrm{~mL}$ Dialysis buffer was taken out of the bag every five minutes, and the same volume release medium at same temperature was added. The concentration of the released drug were calculated by HPLC.

\section{Animal model preparation and group assignment}

Totally, twenty-four health male adult Sprague-Dawley rats (weighting $220 \pm 20 \mathrm{~g}$ ) were provided by Experiment Animal Center of Nanjing University of Chinese Medicine. The rats were all housed in a controlled condition with temperature $\left(18-25^{\circ} \mathrm{C}\right)$ and relative humidity $(65-70 \%)$ on a reverse $12 \mathrm{~h}$ light/dark circle. After acclimating for one week. The rats were randomly divided into 4 groups of 6 rats 
each, that is sham, model, $\mathrm{LN}$ and SH groups. The study protocol was approved by the Ethics Committee of Nanjing University of Chinese Medicine (No.ACU171112).

The PPA model was prepared as previous study reported ${ }^{[26][27]}$. In brief, all rats were fasted for $12 \mathrm{~h}$ but allowed to drink water before the experiment. All surgical procedures were conducted under aseptic conditions. After anesthetized with 1-1.5\% isoflurane, rats were skin prepared and supine position fixed. A $1.5-2 \mathrm{~cm}$ incision was made on the midline of the lower abdomen. The cecum was rubbed smoothly and repeatedly by a file until petechiae appeared on the serosa layer. Then the injured cecum were replaced into the abdominal cavity, and sewed layer by layer. The cecum of rats in sham group were only exposed in the air for $5 \mathrm{~min}$ without rubbing. In the LZ and SH groups, $5 \mathrm{ml} / \mathrm{kg} \mathrm{LN}$ and $0.5 \mathrm{ml} / \mathrm{kg}$ sodium hyaluronate $(\mathrm{SH})$ gel (Baoshilun Furuida Pharmaceutical Co., Ltd.) was sprayed on the injured cecum and surrounding area before closing the abdominal cavity, respectively. All rats were anesthetized with 1-1.5\% isoflurane on the 7th day after operation and an inverted U-incision was used to open the abdominal cavity. The blood samples, and cecum tissues especially adhesive sites were collected for the following analysis. After hemostasis was done completely, the abdominal wall was closed. All rats were then euthanized by sodium pentobarbital ( $200 \mathrm{mg} / \mathrm{kg})$.

\section{Adhesion grading and evaluation}

The degree of adhesion was determined using five-stage adhesion score system ${ }^{[13][28][29]}$ by two independent investigators in a blinded way. This scoring system included five stages adhesion scores range from 0 to 4 . Grade 0 represents no adhesion areas; grade 1 represents $0-25 \%$ zones with thin, avascular and transparent adhesion, indicating a milder inflammatory response; grade 2 represents 25$75 \%$ zones with thick, avascular and opaque adhesion, meaning a mild inflammatory reaction; grade 3 represents $50-75 \%$ thick, capillaries, opaque adhesion, and sharp dissection required, indicating a moderate response; grade 4 represents $75-100 \%$ thick, opaque adhesion with large vessels, and sharp dissection required, meaning a severe reaction.

\section{Masson's trichrome (MT) staining}

A portion of the cecum specimens were placed in $10 \%$ formaldehyde for $24 \mathrm{~h}$, and dehydrated using graded ethanol. Then, the tissues were embedded in paraffin, cut into $4 \mu \mathrm{m}$ thick sections. After the sections were heated and dewaxed, they were stained with MT (Leagene Biotechnology, Beijing) according to routine protocols. Different microscope fields were chosen randomly by microscope (DM2500; Leica, Germany) to evaluate the inflammation.

\section{Enzyme-linked immunosorbent assay (ELISA)}

Blood and tissue samples were harvested respectively. The cecum tissue were stored in $-80^{\circ} \mathrm{C}$ for the following analysis. The blood samples were centrifuged at $3000 \mathrm{rpm}$ for $20 \mathrm{~min}$. Afterward, the supernatant were collected and stored in $4^{\circ} \mathrm{C}$ for the subsequent analysis. The concentrations of IFN- $\gamma$, IL-12, IL-4 and IL- 6 in the cecum tissue and serum were determined by ELISA kit (JinYiBai, Nanjing) 
according to the instructions. The optical density values with a wavelength of $45 \mathrm{~nm}$ were determined by the enzymatic analyzer (Tecan Integral F50, Swiss).

\section{Western blotting (WB)}

Protein from cecum tissues were extracted by a lysis buffer. The concentration of proteins were measured by BCA kit (Beyotime, Shanghai). The protein were separated by sodium dodecyl sulfate-polyacrylamide gel electrophoresis (Bio-Rad, USA). After transferred onto the polyvinylidene fluoride membranes (Millipore, USA), the membranes were blocked in the blocking buffer for $1 \mathrm{~h}$ at $37^{\circ} \mathrm{C}$, incubated with the primary antibodies at $4^{\circ} \mathrm{C}$ overnight. The primary antibodies included anti-TLR4 (1:200 dilution), MyD88 (1:200 dilution), NF-KB (1:200 dilution), $\beta$-actin (1:2000 dilution), which were all provided from Santa Cruz Biotechnology (USA). Then, the membranes were rinsed and incubated with the second antibodies for $80 \mathrm{~min}$ at $37^{\circ} \mathrm{C}$. Finally, the protein expression was imaged by the Chemiluminescence Imaging System (Bio-Rad, USA), and bands were measured by ImageLab Software.

\section{Immunohistochemistry}

After the cecum tissues were fixed in $10 \%$ formaldehyde for $24 \mathrm{~h}$ and embedded in paraffin wax, the tissues were cut into sections. These sections were developed using Diaminobenzidine tetrahydrochloride (DAB) kits (CWBIO, Beijing). The sections of 4- $\mu \mathrm{m}$ thickness were incubated with NF-KB (1:200 dilution) overnight at $4^{\circ} \mathrm{C}$. Second antibody and color were all conducted according to the DAB kits' instruction. Views were randomly visualized under a microscope (DM2500; Leica, Germany).

\section{Quantitative reverse transcription (qRT-PCR)}

Total RNA from the cecum samples were extracted by Trizol reagent (Invitrogen, USA). Nanodrop 2000 was applied to determine the RNA concentration. Based on the manufacturer's instruction of RevertAid ${ }^{T M}$, CDNA was synthesized in a reverse transcript manner. According to the standard protocol, the qRT-PCR was performed using SYBR ${ }^{T M}$ Green Mater Mix (Thermo, USA). The relative changes of mRNA expression were analyzed by the comparative $\mathrm{Ct}$ method as previously described ${ }^{[30]}$. The primers were presented in Table 1. 
Table 1

the primers used for qRT-PCR

\begin{tabular}{|c|c|}
\hline Gene & Primers Sequence \\
\hline \multirow[t]{2}{*}{ TLR4 } & F 5'-3' TGAATCCCTGCATAGAGGTA \\
\hline & R 5'-3'GACCGTTCTGTCATGGAAGG \\
\hline \multirow[t]{2}{*}{ MyD88 } & F 5'-3'GTAGCCAGCCTCTGAAAC \\
\hline & R 5'-3'AGCCAGGATGATGTCTAC \\
\hline \multirow[t]{2}{*}{ NF-KB } & F 5'-3'AGTTGAGGGGACTTTCCCAGGC \\
\hline & R 5'-3'GATTCGAGTATTAGTTCATGGA \\
\hline \multirow[t]{2}{*}{$\beta$-actin } & F 5'-3'TCCTCACTGAGGCCCCGC \\
\hline & R 5'-3'CTGCCCCATGCCATTCTC \\
\hline
\end{tabular}

\section{Statistical analysis}

All experiments were performed in triplicate. All data were analyzed using SPSS 22.0 and were presented as mean \pm standard deviation. Multiple comparisons were performed using one-way analysis of variance followed by LSD test. A level of $P<0.05$ was regarded as statistically significant.

\section{Results}

\section{Physicochemical characterization of LN}

The drug delivery efficiency of PLA loaded with ligustrazine was determined using two EE and LC indicators, as presented in Fig. 1A. It suggested that LN had good entrapment efficiency and loading capacity. Kinetic study of drug release at $\mathrm{PH}=7.4$ value was shown in Fig. 1B. And the microstructure of ligustrazine was spherical shape with smooth surface (Fig. 1C). The diameter distribution of nanoparticle is about $200 \mathrm{~nm}$. The results indicated that PLA loaded with ligustrazine nanoparticles had good biocompatibility and target delivery, which might be a promising agent for preventing adhesion formation.

\section{LN lessened the formation of PPA in rats}

We found that no rats died after surgery, and no significant difference of weight among four groups. Among the twenty-four rats, two rats in model group and one in SH group might have intestinal obstruction or necrosis due to the cecum appeared dark black. The adhesion scores of all groups was summarized in Fig. 2A. Compared with the sham group, the model group exhibited a severe peritoneal adhesion with a higher adhesion score and grade (Fig. 2B and Fig. 2C). The adhesion score and grade of $\mathrm{LN}$ group was significant lower than that of model groups $(P \otimes 0.05)$. The results suggested that $\mathrm{LN}$ could significantly lessen adhesion formation. 


\section{LN suppressed collagen deposition in PPA rats}

Collagen deposition was one of the important pathological processes followed with the inflammatory reaction during the adhesion formation. Masson staining was used to determine the density of collagen fibers deposition among different groups, as shown in Fig. 2D. We found that the fibrin thickness significantly decreased in LN group in comparison with the model group. Compared with the sham group, there were massive inflammatory cells and collagen fibers in the model group. The findings indicated that LN could suppress collagen fibers accumulation.

\section{LN activated the TLR4/MyD88/NF-KB pathway}

To explore the critical effects of LN on TLR4/MyD88/NF-KB pathway, WB and qRT-PCR were applied. In comparison with the sham group, the protein expression levels of TLR4, MyD88 and NF-KB were significantly upregulated in the model group. After LN treatment, TLR4, MyD88 and NF-KB levels were downregulated, as presented in Fig. 3A. These results were in line with the qRT-PCR (Fig. 3B). Besides, the immunohistochemical analysis of NF-KB in four groups were shown in Fig. $3 \mathrm{C}$. The findings revealed that the LN could lessen adhesion formation by activating the TLR4/MyD88/NF-kB pathway.

\section{LN improved the Th1/Th2 balance in PPA rats}

To further determine LN on the downstream of TLR4/MyD88/NF-KB pathway, the levels of Th1-related cytokines (IFN- $y$, IL-12) and Th2-related cytokines (IL-4, IL-6) in serum and cecum tissues were measured using ELISA, respectively. Compared with the sham group, both Th1- and Th2-related cytokines levels in serum and cecum tissues were elevated in model group. Th2-related cytokines levels were higher than Th1-related cytokines levels. After LN intervention, four cytokines were all decreased. But IL-4 and IL-6 levels were lower than IFN- $y$ and IL-12 levels, as shown in Fig. 4. The results demonstrated that LN could maintain Th1/Th2 balance by TLR4/MyD88/NF-kB pathway in PPA rats.

\section{Discussion}

In present study, we found that ligustrazine nanoparticles can effectively prevent adhesion formation after surgery in rat model. It could reduce collagen fibers accumulation, downregulate the expression levels of TLR4, MyD88 and NF-kB, and maintain Th1/Th2 balance. These findings demonstrated that ligustrazine nanoparticles had effective effects on Th1/Th2 balance by activatingTLR4/MyD88/NF-KB pathway in PPA rats.

Ligustraine is an alkaloid monomer of the plants Chuangxiong which is widely used in a variety of disease, such as cardiovascular disease ${ }^{[31]}$. In recent years, various evidences demonstrated that ligustrazine had anti-oxidant and immunity functions ${ }^{[32]}$. Previously, we had reported that ligustrazine could suppress peritoneal fibrosis so as to prevent adhesion formation ${ }^{[19][20]}$. In order to improve its bioavailability, we used the nanotechnology in current study. Nanoparticles with site-specific delivery property have been widely used as delivery agents in the biology and nanomedicine fields. PLA was 
chosen as the ideal nanoparticle carrier due to its lipophilic, biodegradable and biocompatible behaviours on cancer and other treatment ${ }^{[33][34]}$. PLA loaded with ligustrazine were applied in our study, which filled up the short-term metabolism and rapid absorption of ligustrazine in rodent models effectively. Moreover, the LN had stable and sustained release behavior. LN with fairly uniform diameter and spherical morphology is appropriate and effective for ligustrazine targeting-delivery on injured sites.

Postsurgical adhesion formation following cecal abrasion is triggered from the inflammatory reaction, during which various macrophages and neutrophils are activated and proliferated to the injured sites. These cells are regarded as important leukocytes in abdominal immunity ${ }^{[35]}$. The activated T cells move to abdominal cavity, coordinate chemokine production and leukocyte transportation and home to the adhesion sites ${ }^{[36]}$. T cells and their secreted cytokines and chemokines play pivotal roles in the initiation and development of adhesion formation ${ }^{[37]}$. It was reported that the shifting of Th1 and Th2 contributed to the severity of adhesion ${ }^{[12][37]}$. IFN- $\gamma$ was selectively recruited in the pathogenic tissue response ${ }^{[7]}$. IL-4 as an important Th2 cytokine inhibited the development of Th1 cells. It was reported to involve in the pathogenesis of wound healing ${ }^{[38]}$. Up-regulated expression of IL-6 is found in PPA model ${ }^{[1][39]}$. It is reported that IL- 6 can differentiates naïve CD $4^{+}$a $\beta$ T cells into Th17 cells contributed to the pathophysiologic process of immune disorders ${ }^{[40]}$. IL-12 is produced by activated macrophages. It acts an important roles in the activation and proliferation of T cells ${ }^{[41]}$.

In our previous study, we found that the critical regulatory pathway of TLR4/MyD88/NF-KB according to bioinformatics analysis. And we verified several hub genes including IL-6 on PPA models preliminarily. In this study, we found that the Th1-released IFN- $y$ and IL-12 levels, as well as Th2-released IL-4 and IL-6 levels increased in model group when compared with the sham group. Th2 levels were higher than Th1 levels. After LN intervention, the expression levels of Th1 (IFN-y and IL-12), and Th2 (IL-4 and IL-6) decreased. And Th2 levels were lower than Th1. All evidences suggested that LN could activate TLR4/MyD88/NF-KB pathway, regulate the downstream cytokines expression, induce Th1/Th2 differentiation and maintain Th1/Th2 balance so as to prevent adhesion formation. IL-4 produced by Th2 cells inhibit the production of IL-12 and IFN- $\gamma$ secreted by Th1 cells ${ }^{[42]}$. IL-6 can not only induce IL-4 expression to promote Th2 differentiation but also suppress IFN-y production to inhibit Th1 differentiation ${ }^{[43]}$. Due to the dual roles of IL-4 and IL-6, Th2 cells might play a more important role in the T-cell mediated immunity.

\section{Conclusion}

In conclusion, the crucial finding of our study was that $L N$ could markedly prevent postoperative adhesion formation. The underlying mechanism might be partially related to activation of TLR4/MyD88/NF-KB pathway and balance of Th1/Th2 differentiation. $L N$ can be regarded as a promising agent against postoperative peritoneal adhesion.

\section{Abbreviations}


PPA: postoperative peritoneal adhesion; Th1:T helper 1; Th2:T helper 2; PLA:polylactic acid;

LN:ligustrazine nanoparticles; SH:sodium hyaluronate; EE:entrapment efficiency; LC:loading capacity; HPLC:high performance liquid chromatography; DAB:Diaminobenzidine tetrahydrochloride; qRTPCR:Quantitative reverse transcription; MT:Masson's trichrome; ELISA:Enzyme-linked immunosorbent assay; WB:western blotting.

\section{Declarations}

\section{Availability of data and materials}

The data are available from the corresponding authors on reasonable request.

\section{Consent for publication}

Not applicable.

\section{Competing Interests}

The authors declare no conflict of interests.

\section{Funding}

This work was supported by the National Natural Science Foundation of China (No., 81704084, 81673982 and 81603529), the Science and Technology Projects of Jiangsu Provincial Bureau of Traditional Chinese Medicine (YB2017002 and YB2015002), the Priority Academic Program Development of Jiangsu Higher Education Institutions (PAPD), the Open Projects of the Discipline of Chinese Medicine of Nanjing University of Chinese Medicine Supported by the Subject of Academic priority discipline of Jiangsu Higher Education Institutions (NO.ZYX03KF63), the advantages of nursing discipline project of Jiangsu province (2019YSHL005), the Jiangsu Government Scholarship for Overseas Studies, China Scholarship Council and Qing Lan Project. The funders had no roles in the design, data collection and analysis, decision to publish, and preparation of the manuscript.

\section{Authors' contributions}

LLY and YYB conceived and designed the study. ZYL helped the experiment. BZ and ZJL conducted the statistical analysis. WLL and LZ revised the manuscript. All authors read and approved the final manuscript. 


\section{Acknowledgements}

Not applicable.

\section{References}

1. Uyama N, Tsutsui H, Wu S, et al. Anti-interleukin-6 receptor antibody treatment ameliorates postoperative adhesion formation. Sci Rep. 2019;9:17558.

2. Vrijland WW, Jeekel J, van Geldorp HJ, et al. Abdominal adhesions: intestinal obstruction, pain, and infertility. Surg Endosc. 2003;17:1017-22.

3. Schnuriger B, Barmparas G, Branco BC, et al. Prevention of postoperative peritoneal adhesions: a review of the literature. Am J Surg. 2011;201:111-21.

4. Ten BRP, Issa Y, van Santbrink EJ, et al. Burden of adhesions in abdominal and pelvic surgery: systematic review and met-analysis. BMJ. 2013;347:f5588.

5. Awonuga AO, Belotte J, Abuanzeh S, et al. Advances in the Pathogenesis of Adhesion Development: The Role of Oxidative Stress. Reprod Sci. 2014;21:823-36.

6. Koninckx PR, Gomel V, Ussia A, et al. Role of the peritoneal cavity in the prevention of postoperative adhesions, pain, and fatigue. Fertil Steril. 2016;106:998-1010.

7. Tzianabos AO, Holsti MA, Zheng XX, et al. Functional Th1 cells are required for surgical adhesion formation in a murine model. J Immunol. 2008;180:6970-6.

8. Ozbilgin $\mathrm{K}$, Uner MA, Ozkut M, et al. The effects of pirfenidone on Thelper cells in prevention of intraperitoneal adhesions. Kaohsiung J Med Sci. 2017;33:271-6.

9. Harrington LE, Hatton RD, Mangan PR, et al. Interleukin 17-producing CD4 + effector T cells develop via a lineage distinct from the T helper type 1 and 2 lineages. Nat Immunol. 2005;6:1123-32.

10. Buttenschoen K, Fathimani K, Buttenschoen DC. Effect of major abdominal surgery on the host immune response to infection. Curr Opin Infect Dis. 2010;23:259-67.

11. Ishikawa $\mathrm{M}$, Nishioka $\mathrm{M}$, Hanaki $\mathrm{N}$, et al. Perioperative immune responses in cancer patients undergoing digestive surgeries. World J Surg Oncol. 2009;7:7.

12. Chung DR, Chitnis T, Panzo RJ, et al. CD4 + T cells regulate surgical and postinfectious adhesion formation. J Exp Med. 2002;195:1471-8.

13. Bian YY, Yang LL, Yan Y, et al. Identification of candidate biomarkers correlated with pathogenesis of postoperative peritoneal adhesion by using microarray analysis. World J Gastrointest Oncol. 2020;12:54-65.

14. Arung W, Meurisse M, Detry O. Pathophysiology and prevention of postoperative peritoneal adhesions. World J Gastroenterol. 2011;17:4545-53.

15. Wu W, Cheng R, Das NJ, et al. Advances in biomaterials for preventing tissue adhesion. J Control Release. 2017;261:318-36. 
16. Zhou Y, Zhang L, Zhao W, et al. Nanoparticle-mediated delivery of TGF-beta1 miRNA plasmid for preventing flexor tendon adhesion formation. Biomaterials. 2013;34:8269-78.

17. Yoshizawa $H$, Morishita $Y$, Watanabe $M$, et al. TGF-beta(1)-siRNA delivery with nanoparticles inhibits peritoneal fibrosis. Gene Ther. 2015;22:333-40.

18. Cheng $L, M a H$, Shao M, et al. Synthesis of folatechitosan nanoparticles loaded with ligustrazine to target folate receptor positive cancer cells. Mol Med Rep. 2017;16:1101-8.

19. Zhang H, Li D, Li Z, et al. Effect of Ligustrazine on rat peritoneal mesothelial cells treated with lipopolysaccharide. Ren Fail. 2016;38:961-9.

20. Zhang H, Song Y, Li Z, et al. Evaluation of ligustrazine on the prevention of experimentally induced abdominal adhesions in rats. Int J Surg. 2015;21:115-21.

21. Ge H, Lin P, Luo T, et al. Fabrication of Ligusticum chuanxiong polylactic acid microspheres: A promising way to enhance the hepatoprotective effect on bioactive ingredients. Food Chem. 2020;317:126377.

22. Georgiopoulos $P$, Kontou E, Meristoudi A, et al. Tauhe effect of silica nanoparticles on the thermomechanical properties and degradation behavior of polylactic acid. J Biomater Appl. 2014;29:662-74.

23. Zeng L, Mao C, Lu T, et al. Preparation technology and quality assay of ligustrazine poly lactic acid nanoparticles. Chinese Tradit Pat Med. 2013;35:261-3.

24. Mao C, Lu T, Qian L, et al. Study on the preparation of ligustrazine poly lactic acid nanoparticles using orthogonal experiment methods. Chinese Tradit Pat Med. 2013;35:1792-4.

25. Ayumi NS, Sahudin S, Hussain Z, et al. Polymeric nanoparticles for topical delivery of alpha and beta arbutin: preparation and characterization. Drug Deliv Transl Res. 2019;9:482-96.

26. Zhang $\mathrm{H}$, Song $\mathrm{Y}$, Li Z, et al. Evaluation of breviscapine on prevention of experimentally induced abdominal adhesions in rats. Am J Surg. 2016;211:1143-52.

27. Zhao M, Bian YY, Yang LL, et al. HuoXueTongFu Formula Alleviates Intraperitoneal Adhesion by Regulating Macrophage Polarization and the SOCS/JAK2/STAT/PPAR-gamma Signalling Pathway. Mediat Inflamm. 2019;2019:1769374.

28. Kocak I, Unlu C, Akcan Y, et al. Reduction of adhesion formation with cross-linked hyaluronic acid after peritoneal surgery in rats. Fertil Steril. 1999;72:873-8.

29. Kennedy R, Costain DJ, McAlister VC, et al. Prevention of experimental postoperative peritoneal adhesions by N,O-carboxymethyl chitosan. Surgery. 1996;120:866-70.

30. Livak KJ, Schmittgen TD. Analysis of relative gene expression data using real-time quantitative PCR and the 2(-Delta Delta C(T)) Method. Methods. 2001;25:402-8.

31. Shao H, Zhao L, Chen F, et al. Efficacy of Ligustrazine Injection as Adjunctive Therapy for Angina Pectoris: A Systematic Review and Meta-Analysis. Med Sci Monit. 2015;21:3704-15.

32. Guo L, Wang A, Sun $Y$, et al. Evaluation of antioxidant and immunity function of tetramethylpyrazine phosphate tablets in vivo. Molecules. 2012;17:5412-21. 
33. Calzoni E, Cesaretti A, Polchi A, et al. Biocompatible Polymer Nanoparticles for Drug Delivery Applications in Cancer and Neurodegenerative Disorder Therapies. J Funct Biomater 2019;10.

34. Rancan F, Papakostas D, Hadam S, et al. Investigation of polylactic acid (PLA) nanoparticles as drug delivery systems for local dermatotherapy. Pharm Res. 2009;26:2027-36.

35. Jackson PG, Evans SR. Intraperitoneal macrophages and tumor immunity: A review. J Surg Oncol. 2000;75:146-54.

36. de Barros IBL, Malvezzi H, Gueuvoghlanian-Silva BY, et al. Corrigendum to 'What do we know about regulatory T cells and endometriosis? A systematic review'. J Reprod Immunol. 2017;121:34.

37. Chegini N. Peritoneal molecular environment, adhesion formation and clinical implication. Front Biosci. 2002;7:e91-115.

38. Salmon-Ehr V, Ramont L, Godeau G, et al. Implication of interleukin-4 in wound healing. Lab Invest. 2000;80:1337-43.

39. Holmdahl L, Ivarsson ML. The role of cytokines, coagulation, and fibrinolysis in peritoneal tissue repair. Eur J Surg. 1999;165:1012-9.

40. Narazaki M, Kishimoto T. The Two-Faced Cytokine IL-6 in Host Defense and Diseases. Int J Mol Sci 2018;19.

41. Scott P. IL-12: initiation cytokine for cell-mediated immunity. Science. 1993;260:496-7.

42. Narsale A, Moya R, Davies JD. Human CD4(+) CD25(+) CD127(hi) cells and the Th1/Th2 phenotype. Clin Immunol. 2018;188:103-12.

43. Diehl S, Rincon M. The two faces of IL-6 on Th1/Th2 differentiation. Mol Immunol. 2002;39:531-6.

\section{Figures}

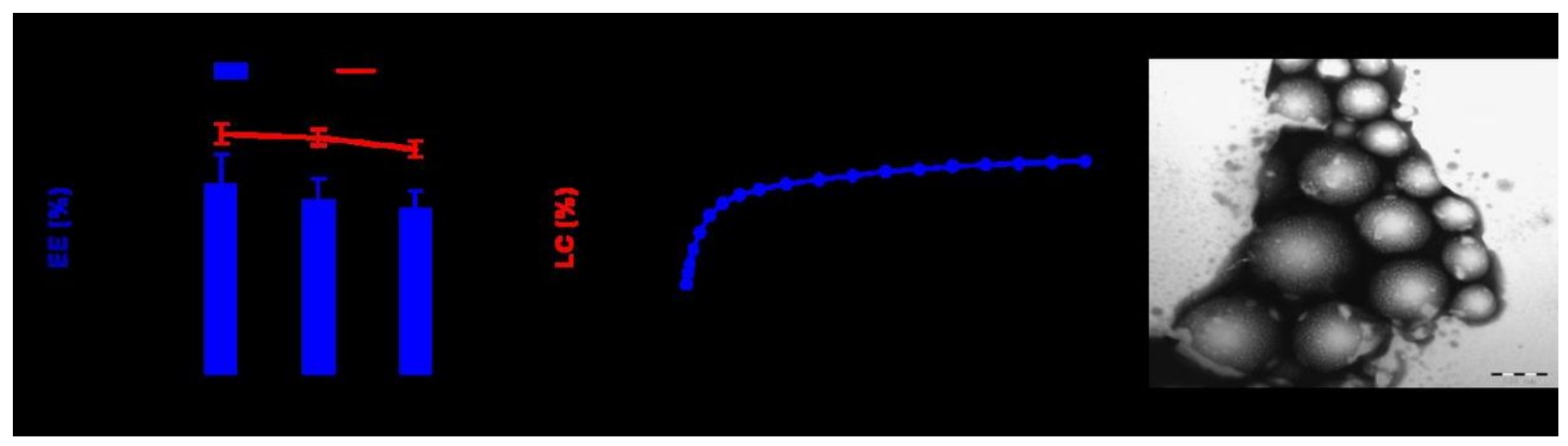

Figure 1

Physicochemical characterization of LN. (A) Entrapment efficiency and loading capacity of LN. (B) Cumulative release curve of $L N$ at $P H=7.4$. (C) Scanning electron microscope image of $L N(\times 200 \mathrm{~nm})$. 


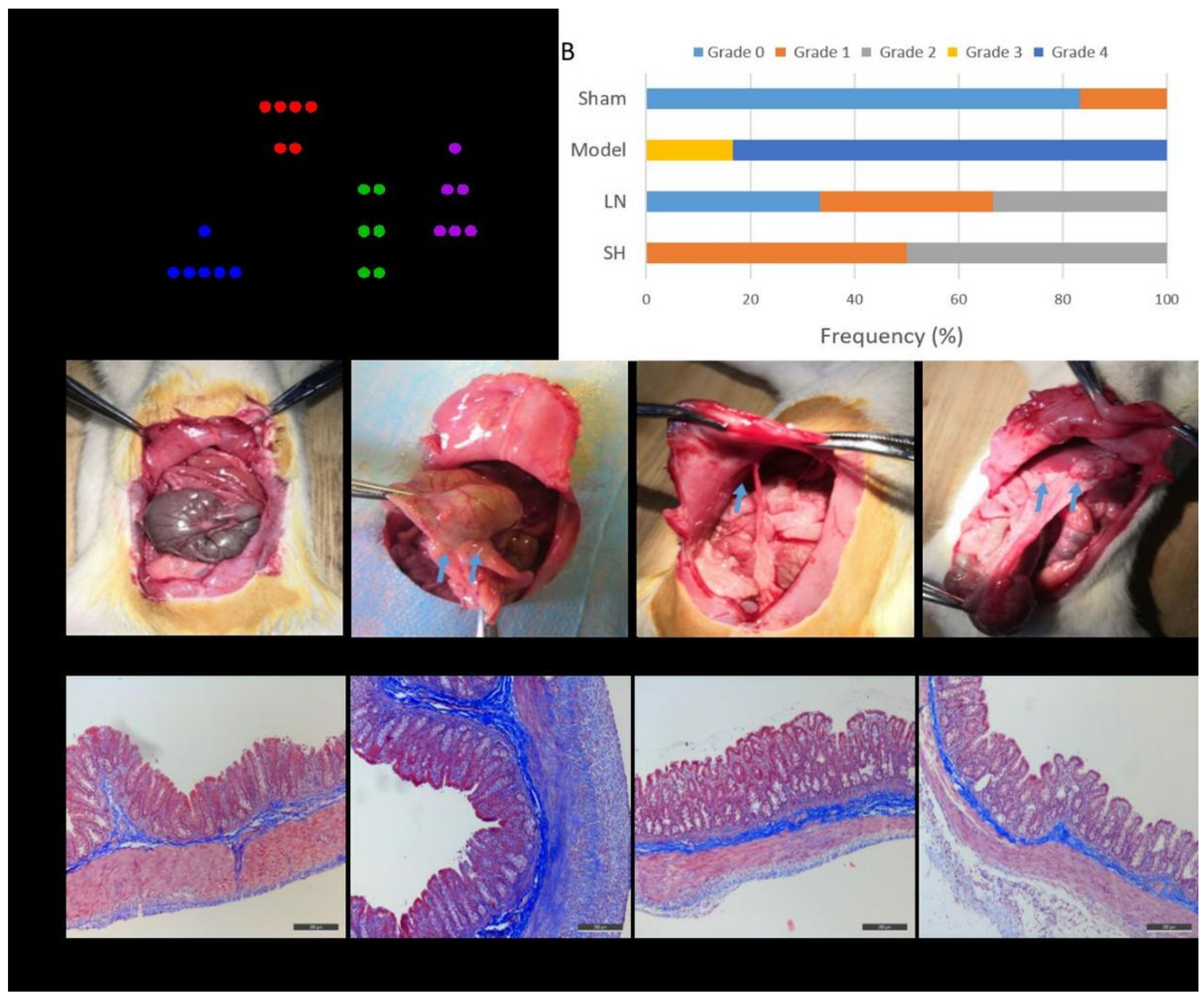

Figure 2

Effect of LN on reducing adhesion formation. $\mathrm{BA}$ Adhesion score of four groups. (B) Frequency of different grades in four groups. (C) Representative images of adhesion formation of different groups. (D) Representative images of Masson's trichrome staining of different groups (100x). 


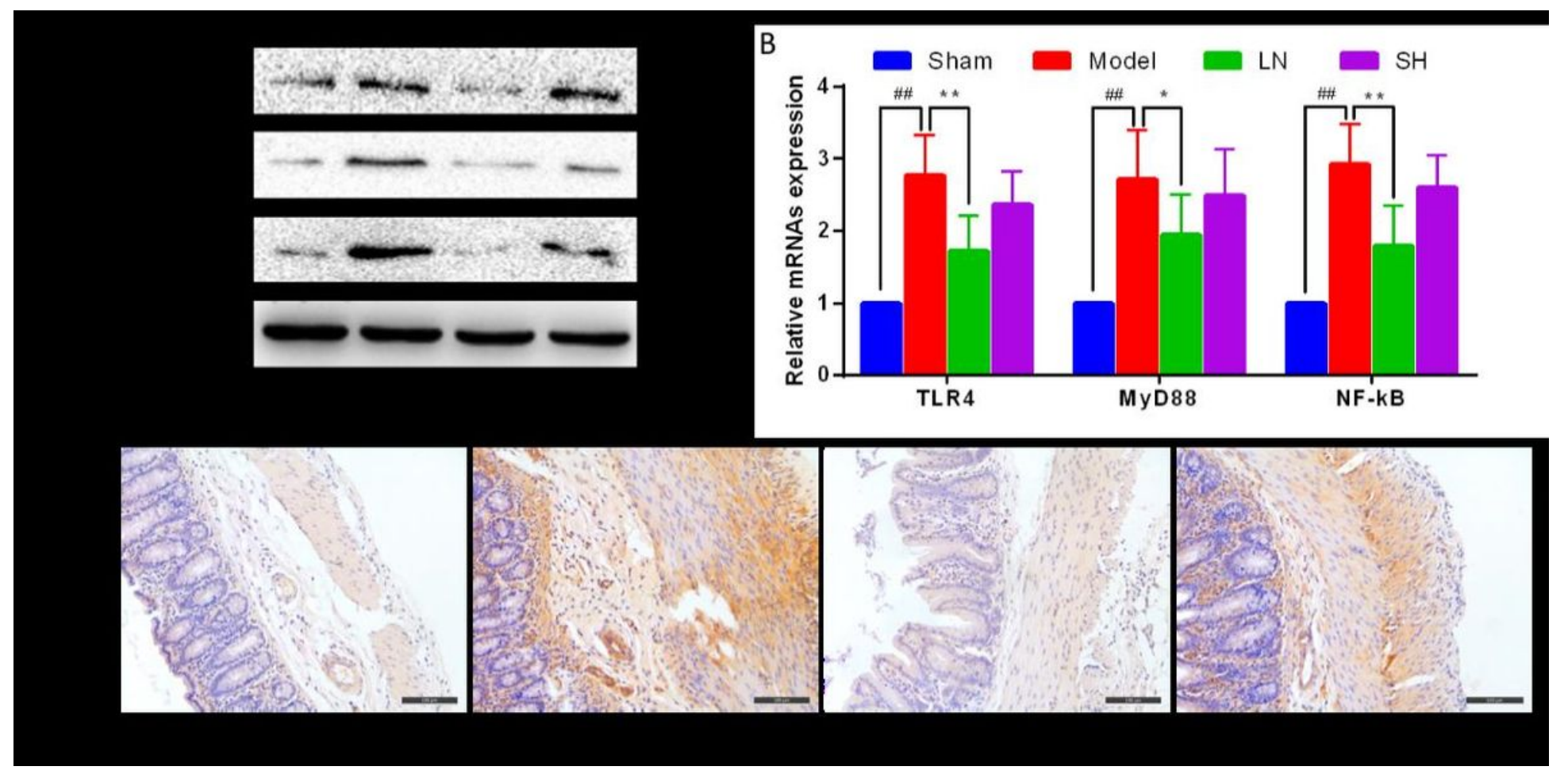

Figure 3

Effect of LN on activating the TLR4/MyD88/NF-KB pathway. (A) The expression levels of TLR4, MyD88 and NF-kB by western Blot. (B) The expression levels of mRNAs by qRT-PCR assay. (C) Representative images of immunohistochemical staining of NF-KB in different groups (200x).

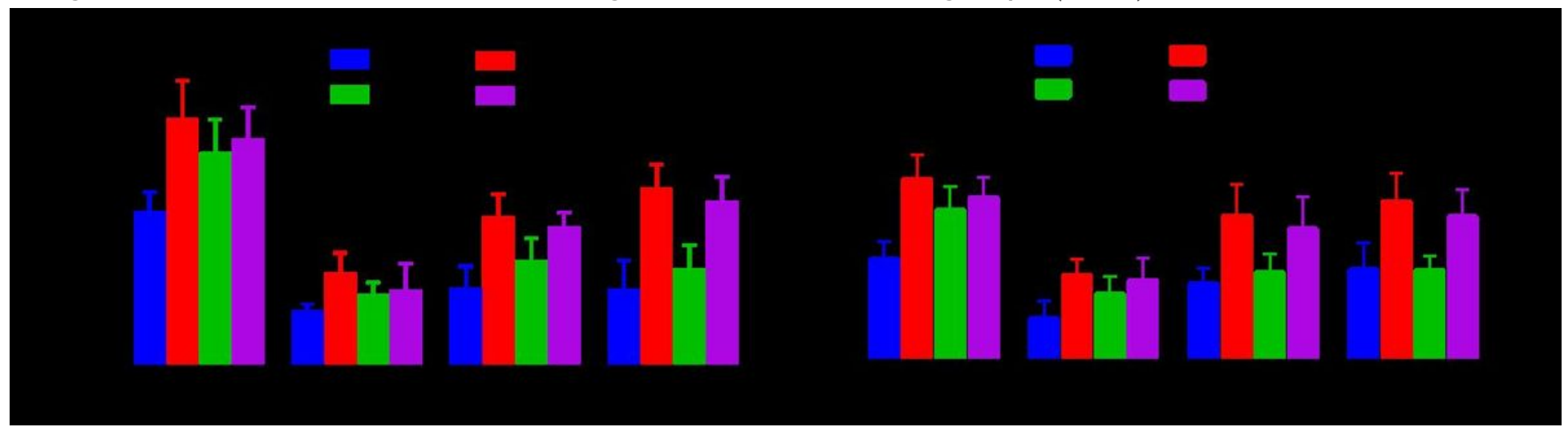

\section{Figure 4}

Effect of LN on improving the Th1/Th2 balance. The levels of Th1-related cytokines (IFN-ץ, IL-12) and Th2-related cytokines (IL-4, IL-6) in the serum and cecum tissues by ELISA assay

\section{Supplementary Files}

This is a list of supplementary files associated with this preprint. Click to download.

- ARRIVEGuidelinesChecklist.pdf 